\title{
Large-scale atomistic simulations of nanostructured materials based on divide-and-conquer density functional theory
}

\author{
F. Shimojo ${ }^{1}$, S. Ohmura ${ }^{1}$, A. Nakano ${ }^{2}$, R. K. Kalia ${ }^{2}$, and P. Vashishta ${ }^{2}$ \\ ${ }^{1}$ Department of Physics, Kumamoto University, Kumamoto 860-8555, Japan \\ ${ }^{2}$ Collaboratory for Advanced Computing and Simulations, Department of Physics \& Astronomy, \\ Department of Computer Science, Department of Materials Science \& Engineering, \\ University of Southern California, Los Angeles, CA 90089-0242, USA
}

\begin{abstract}
A linear-scaling algorithm based on a divide-and-conquer (DC) scheme is designed to perform large-scale molecular-dynamics simulations, in which interatomic forces are computed quantum mechanically in the framework of the density functional theory (DFT). This scheme is applied to the thermite reaction at an $\mathrm{Al} / \mathrm{Fe}_{2} \mathrm{O}_{3}$ interface. It is found that mass diffusion and reaction rate at the interface are enhanced by a concerted metal-oxygen flip mechanism. Preliminary simulations are carried out for an aluminum particle in water based on the conventional DFT, as a target system for large-scale DC-DFT simulations. A pair of Lewis acid and base sites on the aluminum surface preferentially catalyzes hydrogen production in a low activation-barrier mechanism found in the simulations.
\end{abstract}

\section{Introduction}

The reactivity of nano-scale materials is known to differ drastically from their micron-scale counterparts. For example, flame propagation speeds approach $\mathrm{km} / \mathrm{s}$ on the combustion of nanothermites, such as $\mathrm{Al} / \mathrm{Fe}_{2} \mathrm{O}_{3}$ [1] and $\mathrm{Al} / \mathrm{MoO}_{3}$ [2], when the size of $\mathrm{Al}$ nanoparticles is reduced to below $100 \mathrm{~nm}$, in contrast to $\mathrm{cm} / \mathrm{s}$ for traditional thermites. Such peculiar reactive behavior of nanothermites cannot be explained by conventional mechanisms based on mass diffusion of reactants.

Since its microscopic mechanism may be difficult to obtain from experiments, it is desirable to study the reaction of nanoenergetic materials by first-principles simulations. However, large spatial scales are necessary to capture nanostructural effects, while quantummechanical accuracy is required to describe chemical reactions. Recent developments in linear-scaling density functional theory (DFT) [3-6] have set the stage for such large first-principles molecular dynamics (MD) simulation. We have designed divide-and-conquer DFT (DC-DFT) based MD simulations [3, 6] to study the thermite reaction at an $\mathrm{Al} / \mathrm{Fe}_{2} \mathrm{O}_{3}$ interface. The simulations reveal that the rate of redox reactions are enhanced by a concerted metal-oxygen flip mechanism, which leads to two-stage reactions. Rapid initial reaction due to collective metal-oxygen flips is followed by slower reaction based on uncorrelated diffusive motions. These findings may explain recent experimental observation mentioned above.
Hydrogen production by metal particles in water could provide a renewable energy cycle $[7,8]$, if its reaction kinetics is accelerated. Unfortunately, conventional metal-water reaction kinetics is not fast enough to make it viable. As a target system for largescale DC-DFT simulations, we have next selected metal/water systems to suggest a possible nanotechnology-based solution to this problem. Preliminary MD simulations based on the conventional DFT have been carried out to investigate processes of the hydrogen production from water by a cluster (or superatom) consisting of a magic number of aluminum atoms, $\mathrm{Al}_{n}$ (for instance, $n=12$ or 17) [9]. In a low activation-barrier mechanism found in the simulations, a pair of Lewis acid and base sites on the $\mathrm{Al}_{n}$ surface preferentially catalyzes hydrogen production.

\section{Divide-and-conquer density functional theory}

A divide-and-conquer density-functional (DC-DFT) algorithmic framework is developed based on data locality principles to design linear-scaling algorithms for a wide range of scientific applications with tight error control. In DC algorithms [3], spatially localized subproblems are solved in a global embedding field, which is efficiently computed with tree-based algorithms. The three-dimensional space $\Omega$ is covered with overlapping domains $\Omega_{\alpha}$. Each $\Omega_{\alpha}$ is further decomposed into the 
non-overlapping core $\Omega_{0 \alpha}$ and the buffer layer $\Gamma_{\alpha}$. The essential approximation in the DC-DFT algorithm is the replacement of the self-consistent Kohn-Sham Hamiltonian $H$ by its subspace approximation $H_{\alpha}$ for $\Omega_{\alpha}$. The Kohn-Sham equations are solved in each domain $\Omega_{\alpha}$ to obtain locally orthonormal Kohn-Sham orbitals $\left\{\psi_{n}{ }^{\alpha}\right\}$. Boundary conditions on $\left\{\psi_{n}^{\alpha}\right\}$ are imposed at the domain boundary $\partial \Omega_{\alpha}$. We use either the rigid-wall boundary condition or the periodic boundary condition. The global electron dnesity $\rho(r)$ is obtained from $\left\{\psi_{n}{ }^{\alpha}\right\}$ within the non-overlapping core $\Omega_{0 \alpha}$ so that the contaminated wave function values do not contribute to $\rho(r)$. To determine the number of occupied local Kohn-Sham orbitals in the DC-DFT algorithm, the Fermi energy $\varepsilon_{\mathrm{F}}$ is determined globally from the number of valence electrons. The forces acting on atoms are calculated from the Hellmann-Feynman theorem.

For efficient parallel implementation of DFT, we have developed a hierarchical real-space grid method based on higher-order finite differencing [10], and multigrid acceleration [11]. In the hierarchical grid method, a realspace multigrid is adaptively refined near each atom to accurately operate the ionic pseudopotentials [12] on the electronic wave functions. The energy functional is iteratively minimized using a preconditioned conjugategradient method [13]. The hierarchical grid DC-DFT algorithm has been implemented on parallel computers based on spatial decomposition. In this parallelization scheme, each domain is local to a processor, and the Kohn-Sham orbitals need not be exchanged between processors. Consequently, there is no need for the massive communication required for orthonormalization in conventional parallel DFT algorithms.

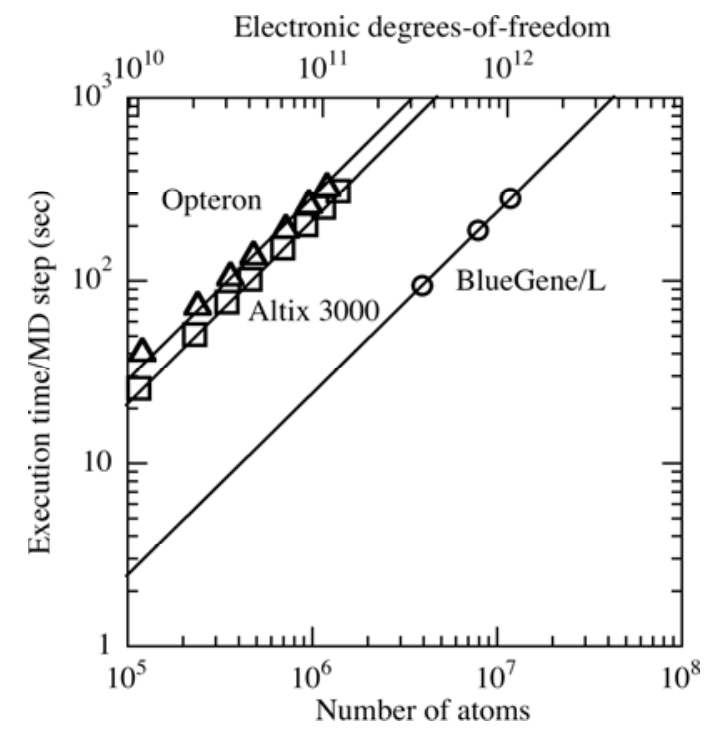

Fig. 1. I Benchmark tests of the DC-DFT MD algorithm on 131,072 BlueGene/L processors (circles), 1,920 Itanium2 processors of Altix 3000 at NASA (squares), and 2,000 Opteron processors (triangles). The execution time per MD step is shown as a function of the number of atoms, and lines show $O(N)$ scaling [6].

The parallel DC-DFT program is written in Fortran 90 with MPI (Message Passing Interface) for massage passing. Scalability tests of the parallel DC-DFT algorithm have been performed on a wide range of platforms, such as the 131,072-processor IBM BlueGene/L at the Lawrence Livermore National Laboratory. Figure 1 shows the execution time of the DCDFT algorithm per MD step as a function of the number of atoms for alumina systems on three platforms [6]. The execution time scales linearly with the number of atoms. The largest benchmark test in this study involves $11,796,480$ atoms $(1,035,825,315,840$ electronic degreesof-freedom) on 131,072 BlueGene/L processors.

\section{Thermite reactions}

\subsection{Details of calculation}

In our DC-DFT based MD simulations, interatomic forces are computed quantum mechanically in the framework of DFT within a generalized gradient approximation (GGA) [14]. As described in the previous section, electronic wave functions are represented on a real-space grid [10], which is augmented with a coarse multigrid [11] to accelerate the convergence of iterative solutions. The DC-DFT algorithm has well-defined parameters to control the data locality, with which the solutions converge rapidly. Also the total energy is well conserved during the MD simulation [6].

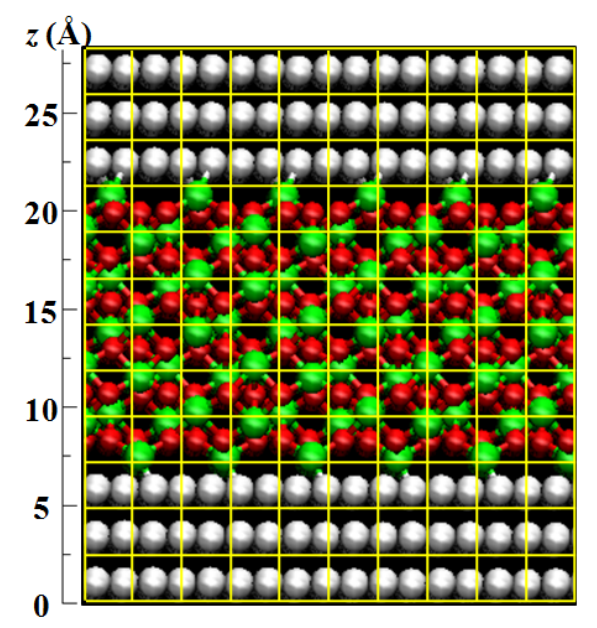

Fig. 1. Initial atomic configuration. Meshes show the nonoverlapping cores used by the DC-DFT method. Two interfacial regions are defined as slabs with a thickness of $4 \AA$ parallel to the $x y$ plane. Length scale in angstrom is marked in the $\mathrm{z}$ direction.

The simulated system is a stack of $\mathrm{Al}$ and $\mathrm{Fe}_{2} \mathrm{O}_{3}$ layers involving $1152\left(144 \mathrm{Fe}_{2} \mathrm{O}_{3}+432 \mathrm{Al}\right)$ atoms in an orthorhombic supercell with dimensions $20.1 \times 26.2 \times$ $28.2 \AA$ with periodic boundary conditions. The hematite $\left(\mathrm{Fe}_{2} \mathrm{O}_{3}\right)$ crystal, cut along (0001) planes to expose $\mathrm{Fe}$ planes, is placed in the supercell with the (0001) direction parallel to the $\mathrm{z}$ direction. The Fe planes of the hematite are attached to (111) planes of the face-centered cubic $\mathrm{Al}$ crystal at the two interfaces. The grid spacing $\sim 0.25 \AA$ (corresponding to the cutoff energy of $45 \mathrm{Ry}$ in the planewave-based method) is sufficiently small to obtain a good convergence of the total energy. The DC-DFT method 
divides the system into $960(=8 \times 10 \times 12)$ domains of dimensions $2.51 \times 2.62 \times 2.35 \AA$. Figure 1 shows the side (yz) view of the non-overlapping cores of the domains in the supercell. Each domain is augmented with a buffer layer of depth $\sim 2.2 \AA$ to avoid boundary effects. The interatomic forces thus computed quantum-mechanically are used to integrate Newton's equations of motion numerically (with a time step of 0.84 fs) in MD simulations to study atomic motions and chemical reactions. The MD simulations are carried out at temperature $2000 \mathrm{~K}$ in the canonical ensemble.

\subsection{Enhanced reactivity}

Figure 2 shows snapshots of the atomic configuration. Oxygen atoms in $\mathrm{Fe}_{2} \mathrm{O}_{3}$ migrate into the $\mathrm{Al}$ metal to form aluminum oxide and leaves behind liquid iron. Our DCDFT simulation thus describes complete thermite reaction, $2 \mathrm{Al}+\mathrm{Fe}_{2} \mathrm{O}_{3} \rightarrow \mathrm{Al}_{2} \mathrm{O}_{3}+2 \mathrm{Fe}$.

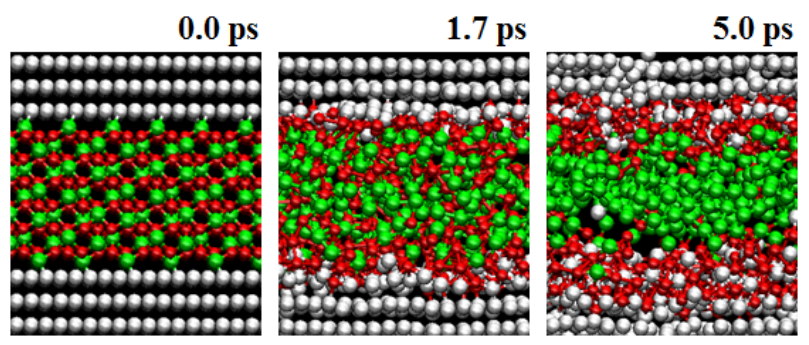

Fig. 2. Snapshots of the atomic configuration.

To investigate the reaction process, we define interfacial regions as slabs with a thickness of $4 \AA$ (i.e. 2 $\AA$ above and below the boundaries) parallel to the $x y$ plane (see figure 1). Mean square displacements of $\mathrm{O}$ atoms along the $\mathrm{z}$ direction are calculated using the simulation data for $0-3 \mathrm{ps}$ as shown in figure 3 . The solid and dashed lines are obtained from $\mathrm{O}$ atoms in the interfacial and Fe-side regions, respectively. Here, the boundary between iron-oxide and aluminum-oxide at each interface is located by calculating the average $\mathrm{z}$ coordinate of $\mathrm{Al}$ atoms facing $\mathrm{Fe}$ atoms. Figure 3 shows that $\mathrm{O}$ atoms in the interfacial region are much more diffusive than those on the $\mathrm{Fe}$ side.

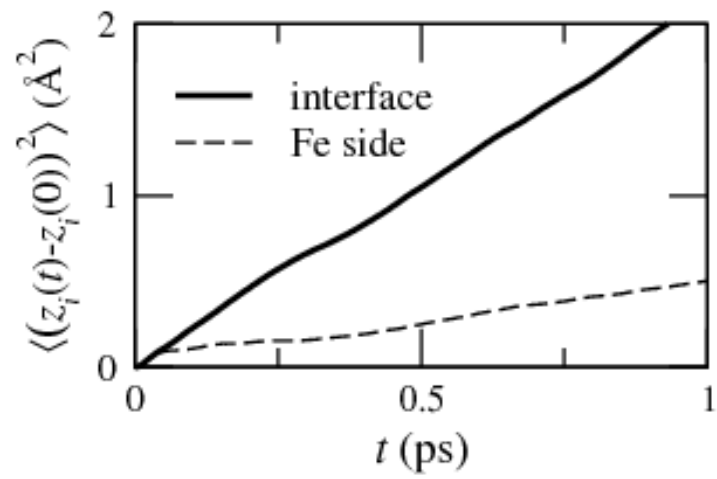

Fig. 3. Mean square displacements of $\mathrm{O}$ atoms along the $\mathrm{z}$ direction are plotted as a function of time. The solid and dashed curves are for $\mathrm{O}$ atoms in the interfacial and $\mathrm{Fe}$-side regions, respectively.

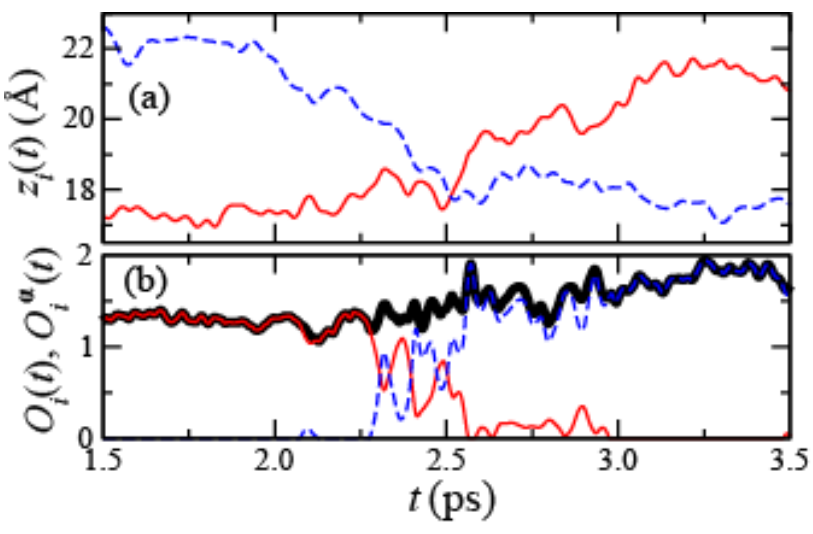

Fig. 4. (a) Time evolution of the $\mathrm{z}$ coordinates of focused $\mathrm{O}$ (solid line) and $\mathrm{Al}$ (dashed line) atoms labelled as ' $\mathrm{O}$ ' and 'Al' in figure 5. (b) Time evolution of the total and partial SBOP, $O_{i}(t)$ and $O_{i}^{\alpha}(t)$, associated with the oxygen atom. The thick, thin, and dashed curves show $O_{i}(t), O_{i}^{\mathrm{Fe}}(t)$ and $O_{i}^{\mathrm{Al}}(t)$, respectively.

To understand the mechanism of the enhanced diffusivity at the interface, we have examined the time evolution of the atomic configuration in the interfacial region and found a concerted metal-oxygen flip mechanism. In this mechanism, $\mathrm{O}$ atoms switch their positions with neighboring $\mathrm{Al}$ atoms while diffusing in the $\mathrm{z}$ direction. A typical example of such events is shown in Fig. 4(a), where the time evolution of the $\mathrm{Z}$ coordinates of the $\mathrm{O}$ and $\mathrm{Al}$ atoms of interest is shown. The $\mathrm{O}$ atom moves upward in concert with the $\mathrm{Al}$ atom moving downward. The switching motion between the $\mathrm{O}$ and $\mathrm{Al}$ atoms is shown in figure 5 .
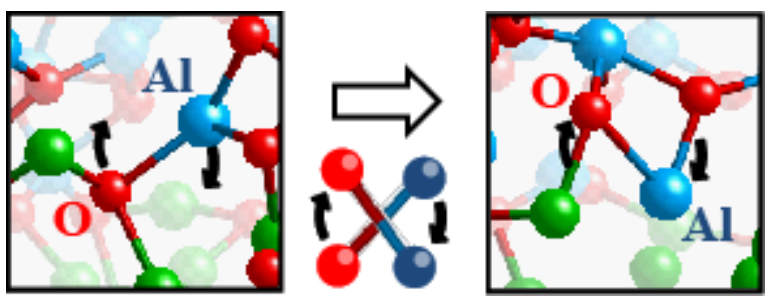

Fig. 5. Atomic configurations near the $\mathrm{O}$ and $\mathrm{Al}$ atoms of interest (labeled as ' $\mathrm{O}$ ' and 'Al') at 2.3 and $2.8 \mathrm{ps}$.

To explain the quantum-mechanical origin of this mechanism, we calculate the bond-overlap population $O_{i j}(t)$ between $i^{\text {th }}$ and $j^{\text {th }}$ atoms as a function of time $t$ [15]. We also define the sum of the bond-overlap population (SBOP) for each oxygen atom: Partial SBOP $O_{i}^{\alpha}(t)$ for the $i^{\text {th }}$ oxygen atom is defined as $O_{i}^{\alpha}(t)=\Sigma_{j \neq \alpha} O_{i j}(t)$, where $\alpha$ is $\mathrm{Fe}$ or $\mathrm{Al}$; and the total SBOP is $O_{i}(t)=O_{i}^{\mathrm{Fe}}(t)+$ $O_{i}^{\mathrm{Al}}(t)$. Figure 4(b) shows $O_{i}(t)$ and $O_{i}^{\alpha}(t)$ associated with the $\mathrm{O}$ atom. For $t<2.3 \mathrm{ps}$, the oxygen atom resides in the iron-oxide region, and $O_{i}^{\mathrm{Fe}}(t)$ has finite values, while $O_{i}^{\mathrm{Al}}(t)$ is nearly zero. At $t \sim 2.3 \mathrm{ps}$, the oxygen atom starts to migrate into the aluminum side and $O_{i}^{\mathrm{Al}}(t)$ begins to increase. For $2.3 \mathrm{ps}<t<2.7 \mathrm{ps}, O_{i}^{\mathrm{Fe}}(t)$ and $O_{i}^{\mathrm{Al}}(t)$ have comparable values while the oxygen atom is moving across the interface. For $t>3.0 \mathrm{ps}, O_{i}^{\mathrm{Fe}}(t)$ becomes zero, while $O_{i}^{\mathrm{Al}}(t)$ converges to a finite value, indicating that the oxygen atom is chemically bonded only with $\mathrm{Al}$ atoms. The switching motion between $\mathrm{O}$ and $\mathrm{Al}$ atoms at 
the interface is thus triggered by the change of chemical bonding associated with these atoms.

To quantify the collective switching motion between $\mathrm{O}$ and $\mathrm{Al}$ atoms, we calculate the correlation function between the displacements of atoms along the $z$ direction:

$$
d_{\mathrm{O}-\mathrm{Al}}(t)=\left\langle\Delta z_{i}(t) \bullet \Delta z_{j}(t)\right\rangle / t^{2},
$$

where $\Delta z_{i}(t)=z_{i}\left(t+t_{0}\right)-z_{i}\left(t_{0}\right)$ with $z_{i}(t)$ being the $z$ coordinate of the $i^{\text {th }}$ ion at time $t$. The brackets mean the average over both the time origin $t_{0}$ and atomic pairs $(i \in$ $\mathrm{O}, j \in \mathrm{Al}$ ). In the calculation, atomic pairs whose distance is less than $2.3 \AA$ at $t_{0}$ are selected. Since we are interested in the correlation between diffusing $\mathrm{O}$ and $\mathrm{Al}$ atoms, we include atomic pairs that satisfy the conditions $\left|\Delta z_{i}(T)\right|>2 \AA$ and $\left|\Delta z_{j}(T)\right|>2 \AA$ at $t=2$ ps. The results reveal negative correlation in $d_{\mathrm{O}-\mathrm{Al}}(t)$ for $t>0.5 \mathrm{ps}$, which reflects the collective switching motion between $\mathrm{O}$ and $\mathrm{Al}$ atoms at the interface as shown in Fig. 6. Such negative correlation does not exist on the Al side, indicating independent diffusive motions of $\mathrm{Al}$ and $\mathrm{O}$ atoms.

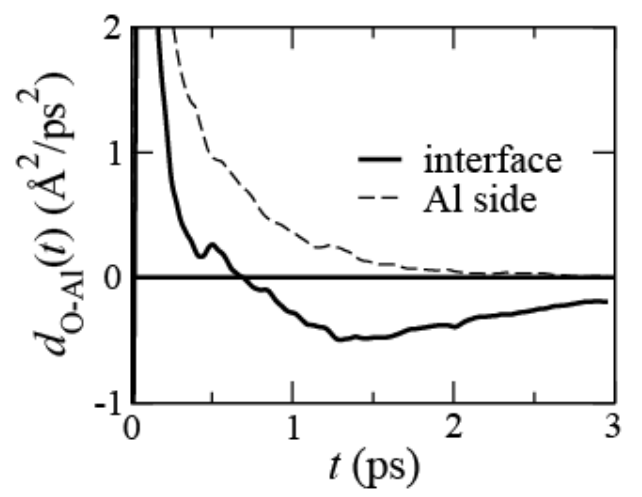

Fig. 6. Negative correlation associated with concerted $\mathrm{Al}$ and $\mathrm{O}$ motions at the interface. Correlation functions between displacements of $\mathrm{O}$ and $\mathrm{Al}$ atoms along the $\mathrm{z}$ direction (defined in Eq. (1)) are shown as a function of time. The solid and dashed curves are obtained in the interfacial and Al-side regions.

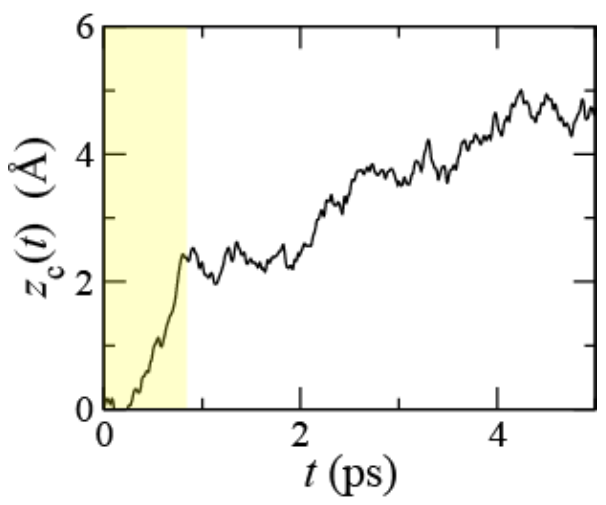

Fig. 7. Two-stage reactions of thermite. Time evolution of the positions $z_{\mathrm{c}}(t)$ of the reaction fronts. The shade $(0<t<1)$ highlights the rapid first-stage reaction due to concerted Al-O motions, which is followed by slow reaction based on uncorrelated diffusion

The enhanced mass diffusivity at the metal-oxide interface leads to a two-stage reaction behavior. The position $z_{\mathrm{c}}(t)$ of the reaction front calculated from the coordinates of oxygen atoms at the forefront of oxidation, as shown in figure 7 . For $t<1 \mathrm{ps}, z_{\mathrm{c}}(t)$ increases rapidly as oxygen atoms migrate into the $\mathrm{Al}$ side, which is accelerated by the collective switching mechanism. This is followed by a slower reaction due to uncorrelated diffusion of atoms. Though slower than the concerted thermite reaction, the second-stage diffusion here could still be faster than conventional diffusion, due to defectmediated giant diffusivity because of the depletion of $\mathrm{O}$ atoms in the near-interface oxides. Such two-stage reactions may be related to the experimental observation in nano-scale thermite.

\section{Hydrogen Production from water using aluminum-cluster catalysts}

As a target system for the next large-scale DC-DFT simulations, we have selected metal/water systems. Here, we describe the results of preliminary simulations for the hydrogen production from water by an aluminium cluster (or superatom) consisting of a magic number of aluminum atoms, $\mathrm{Al}_{n}$ (for instance, $n=12$ or 17) [9]

\subsection{Details of calculation}

The electronic states are calculated using the projectoraugmented-wave (PAW) method [16], which is an allelectron electronic-structure-calculation method within the frozen-core approximation in the framework of DFT. The momentum-space formalism is utilized, where the plane-wave cutoff energies are 30 and $250 \mathrm{Ry}$ for the electronic pseudo-wave functions and the pseudo-charge density, respectively. The energy functional is minimized iteratively using a preconditioned conjugate-gradient method [14]. The $\Gamma$ point is used for Brillouin zone sampling. Projector functions are generated for the $3 s, 3 p$ and $3 d$ states of $\mathrm{Al}$, the $2 s$ and $2 p$ states of $\mathrm{O}$, and the $1 s$ state of $\mathrm{H}$.

MD simulations are carried out at temperatures of 300 , 500 and $1000 \mathrm{~K}$ in the canonical ensemble with a time step of 11 a.u. $(\sim 0.264 \mathrm{fs})$. The system contains an $\mathrm{Al}_{17}$ cluster and $84 \mathrm{H}_{2} \mathrm{O}$ molecules (in total of 269 atoms) in a box of dimensions $12.58 \times 12.58 \times 18.87 \AA^{3}$ (see figure 8 ). The system size is determined from the density of water in ambient conditions, and periodic boundary conditions are imposed.

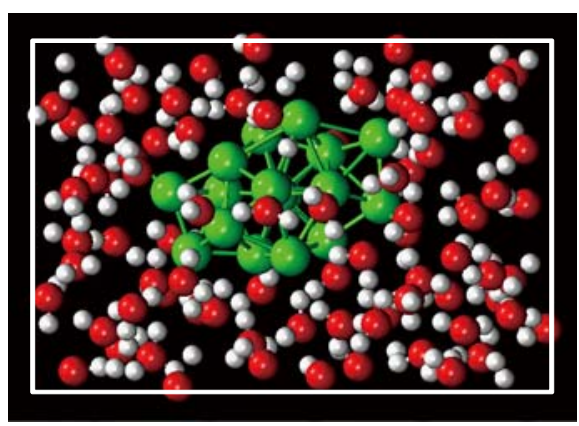

Fig. 8. Snapshot of the $\mathrm{Al}_{17}+$ water system. 


\subsection{Low activation-barrier mechanism of hydrogen production}

In simulations at room temperature $(300 \mathrm{~K})$, six water molecules bond to the Al clusters. Formation of these Al$\mathrm{O}$ bonds enhances the Lewis-base character of $\mathrm{Al}$ atoms that are not connected to the water molecules, thereby preventing further bonding of water molecules to the $\mathrm{Al}$ clusters. Dissociation of water molecules is not observed within the limited simulation time (several ps) at this temperature. Even when the temperature is raised to 500 $\mathrm{K}$, still no water molecule dissociates. The atomistic process of hydrogen production is successfully observed at $1000 \mathrm{~K}$. In total, three hydrogen molecules are produced within $6 \mathrm{ps}$.

Before a hydrogen molecule is produced, a hydrogen atom is adsorbed on the $\mathrm{Al}$ cluster as shown in figure 9. The reaction begins with the dissociation of an $\mathrm{H}_{2} \mathrm{O}$ molecule bonding to an $\mathrm{Al}$ atom, as one of its hydrogen atoms moves toward a neighboring $\mathrm{H}_{2} \mathrm{O}$ molecule to form a hydronium ion $\left(\mathrm{H}_{3} \mathrm{O}^{+}\right)$. This is followed by a chain of hydrogen bond switching events (denoted by arrows in figure 9(a)) that involves in total of four $\mathrm{H}_{2} \mathrm{O}$ molecules, and finally a hydrogen atom bonds to an $\mathrm{Al}$ atom after $190 \mathrm{fs}$. This proton transfer is induced by the Lewis acidbase characters of the participating $\mathrm{Al}$ atoms [17]. Although it is hard for the water molecule to dissociate by itself, the Grotthuss mechanism greatly reduces the activation barrier for the dissociation. This process can be written as

$$
\mathrm{Al}-\mathrm{OH}_{2}+3 \mathrm{H}_{2} \mathrm{O}+\mathrm{Al}^{\prime} \rightarrow \mathrm{Al}-\mathrm{OH}+3 \mathrm{H}_{2} \mathrm{O}+\mathrm{Al}^{\prime}-\mathrm{H} .
$$
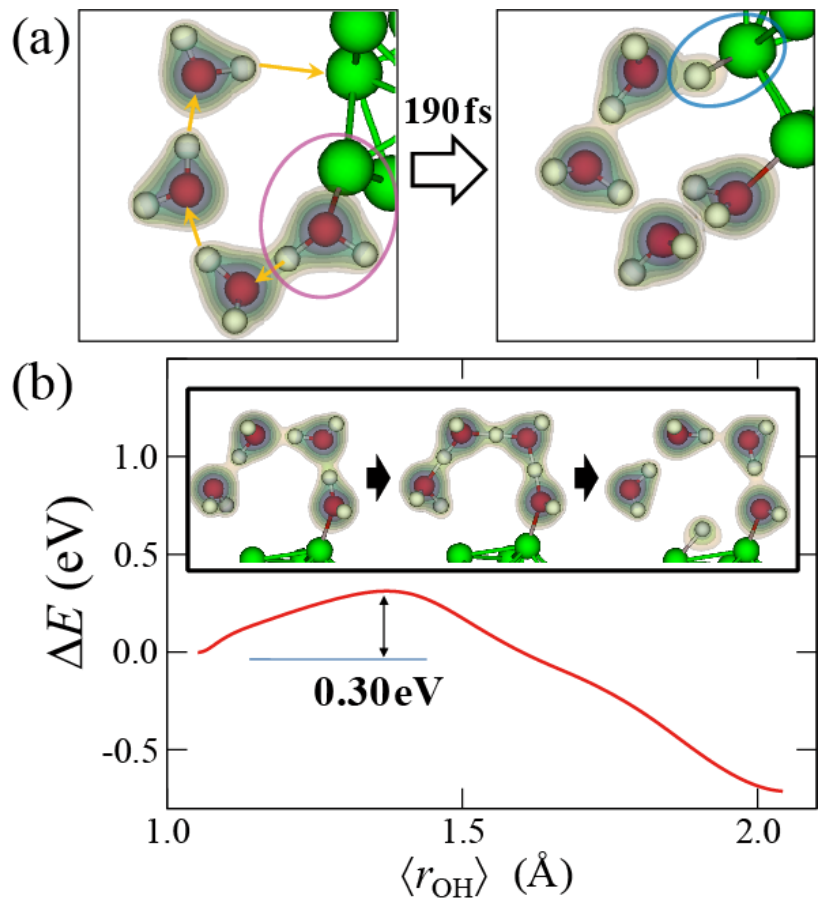

Fig. 9. (a) Adsorption of a hydrogen atom on the Al cluster through the Grotthuss mechanism observed in MD simulation. (b) Energy profile along the reaction path for the production of a hydroxide ion and a hydrogen atom, $\mathrm{Al}+4 \mathrm{H}_{2} \mathrm{O}+\mathrm{Al} \rightarrow \mathrm{Al}-$ $\mathrm{OH}+3 \mathrm{H}_{2} \mathrm{O}+\mathrm{Al}-\mathrm{H}$, obtained by nudged elastic band calculations.
From nudged elastic band calculations [18], the activation energy for this reaction is estimated to be $\Delta=0.3 \mathrm{eV}$ as shown in figure $9(\mathrm{~b})$. Note that the $\mathrm{Al}-\mathrm{OH}$ product of Eq. (2) is quickly converted back to $\mathrm{Al}-\mathrm{H}_{2} \mathrm{O}$ again by the Grotthuss mechanism, which has the activation energy of only $0.03 \mathrm{eV}$.

A hydrogen molecule is produced from the water molecule and the hydrogen atom on the Al cluster as shown in figure 10(a). This hydrogen-production reaction is thus summarized as

$$
\mathrm{Al}-\mathrm{OH}_{2}+\mathrm{Al}-\mathrm{H} \rightarrow \mathrm{Al}-\mathrm{OH}+\mathrm{Al}+\mathrm{H}_{2} \text {. }
$$

The activation energy is estimated to be $\Delta=0.1 \mathrm{eV}$ (see figure $10(\mathrm{~b})$ ), which is lower than that for hydrogen-atom generation of Eq. (2). We also study the finitetemperature effect by calculating the activation free energy [19], which gives a nearly identical value, $\Delta=$ $0.08 \mathrm{eV}$, at $300 \mathrm{~K}$. Since the resulting hydroxide ion of Eq. (3) has a strong Lewis-base character and returns to a water molecule again through the Grotthuss mechanism, the hydrogen production can continue, especially in acidic conditions.
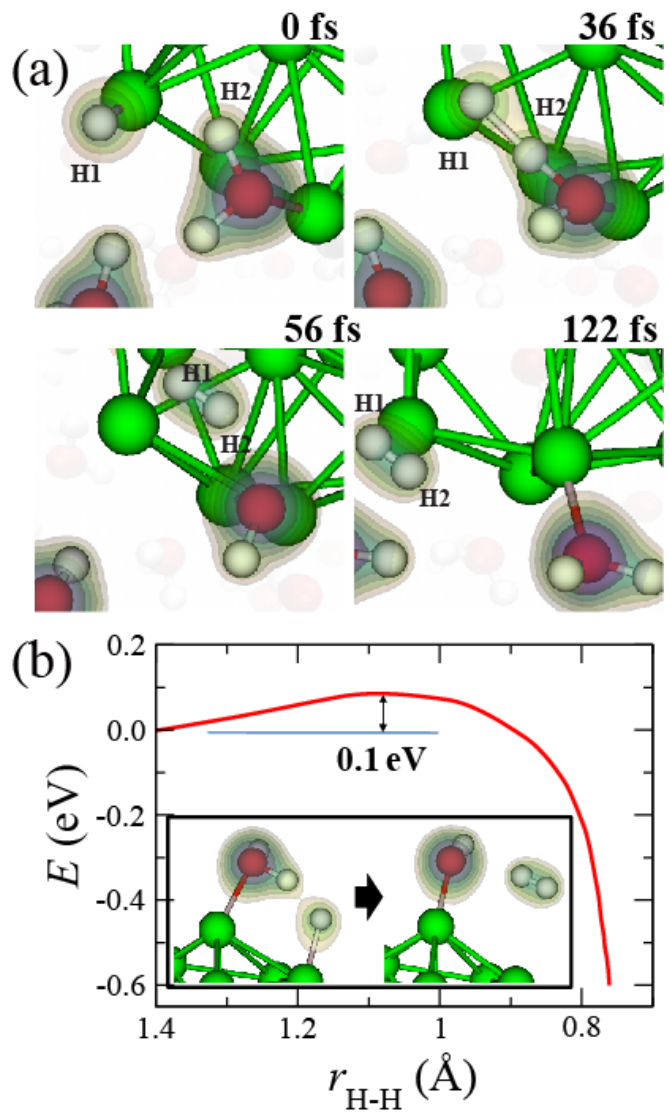

Fig. 10. (a) Hydrogen-production proccess observed in MD simulation. A hydrogen molcule is formed by H1 and H2. (b) Energy profile along the reaction path of molecular hydrogen production, $\mathrm{Al}-\mathrm{OH}_{2}+\mathrm{Al}-\mathrm{H} \rightarrow \mathrm{Al}-\mathrm{OH}+\mathrm{Al}+\mathrm{H}_{2}$, as a function of the distance between the two hydrogen atoms that form a hydrogen molecule, obtained by nudged elastic band calculations. The distribution of the electron density larger than 0.05 a.u. is shown by contour surfaces.

We are currently planning a 10,000-atom DC-DFT based MD simulation for aluminum/water systems to study the effect of acidic conditions. 


\section{Summary}

Density-functional method of electronic-structure calculations has been developed based on the linearscaling divide-and-conquer scheme. Using this scheme, large-scale dynamic simulations become possible with quantum-mechanical accuracy. This method has been applied to investigate the thermite reaction of $\mathrm{Fe}_{2} \mathrm{O}_{3}$ with aluminum. A redox reaction to form iron metal and $\mathrm{Al}_{2} \mathrm{O}_{3}$ initiates with the rapid formation of $\mathrm{Al}-\mathrm{O}$ bonds at the interface within $1 \mathrm{ps}$, followed by the propagation of the combustion front. The concerted metal-oxygen flip mechanism enhances mass diffusion and reaction rate at the interface.

Conventional ab initio molecular dynamics simulation has revealed rapid hydrogen production from water by a cluster (or superatom) consisting of a magic number of aluminum atoms, $\mathrm{Al}_{17}$. We found a low activation-barrier mechanism, in which a pair of Lewis acid and base sites on the $\mathrm{Al}_{n}$ surface preferentially catalyzes hydrogen production. This reaction is immensely assisted by rapid proton transport in water via a chain of hydrogen-bond switching events similar to the Grotthuss mechanism, which converts hydroxide ions to water molecules at the Lewis-acid sites and supplies hydrogen atoms at the Lewis-base sites.

\section{References}

1. K. B. Plantier, M. L. Pantoya, and A. E. Gash, Combust. Flame 140, 299 (2005)

2. B. S. Bockmon et al., J. Appl. Phys. 98, 064903 (2005)

3. W. T. Yang, Phys. Rev. Lett. 66, 1438 (1991)

4. S. Goedecker, Rev. Mod. Phys. 71, 1085 (1999)

5. D. R. Bowler et al., J. Phys.: Condens. Matter 20 , 290301 (2008)

6. F. Shimojo, R. K. Kalia, A. Nakano, and P. Vashishta, Phys. Rev. B 77, 085103 (2008)

7. A. Steinfeld, Sol. Energy 78, 603 (2005).

8. T. Yabe et al., Appl. Phys. Lett. 89, 261107 (2006)

9. F. Shimojo, S. Ohmura, A. Nakano, R. K. Kalia, and P. Vashishta, Phys. Rev. Lett. 104, 126102 (2010)

10. J. R. Chelikowsky, N. Troullier, K. Wu, et al., Phys. Rev. B 50, 11355 (1994)

11. E. L. Briggs, D. J. Sullivan, and J. Bernholc, Phys. Rev. B 54, 14362 (1996)

12. N. Troullier and J. L. Martins, Phys. Rev. B 43, 8861 (1991)

13. F. Shimojo et al., Comput. Phys. Commun. 140, 303 (2001)

14. J. P. Perdew, K. Burke, and M. Ernzerhof, Phys. Rev. Lett. 77, 3865 (1996)

15. F. Shimojo, R. K. Kalia, A. Nakano, and P. Vashishta, Phys. Rev. E 77, 066103 (2008)

16. P. E. Blöchl, Phys. Rev. B 50, 17953 (1994)

17. P. J. Roach et al., Science 323, 492 (2009).

18. G. Henkelman, and H. Jonsson, J. Chem. Phys. 113, 9978 (2000).

19. K. C. Hass et al., Science 282, 265 (1998). 